\title{
TLR2-targeted secreted proteins from Mycobacterium tuberculosis are protective as powdered pulmonary vaccines
}

\author{
Anneliese S. Tyne ${ }^{a}$, John Gar Yan Chan ${ }^{b}$, Erin R. Shanahan a , Ines Atmosukarto ${ }^{\text {, }}$ \\ Hak-Kim Chan $^{\mathrm{b}}$, Warwick J. Britton ${ }^{\mathrm{a}, \mathrm{d}, *}$, Nicholas P. West ${ }^{\mathrm{a}, 1}$ \\ a Tuberculosis Research Program, Centenary Institute, Locked Bag No 6, Newtown, NSW 2042, Australia \\ ${ }^{\mathrm{b}}$ Faculty of Pharmacy, University of Sydney, Sydney, NSW 2006, Australia \\ ${ }^{\mathrm{c}}$ Lipotek Pty Ltd, Research School of Biology, Australian National University, ACT 0200, Australia \\ d Discipline of Medicine, Central Clinical School, Sydney Medical School, University of Sydney, Sydney, NSW 2006, Australia
}

\section{A R T I C L E I N F O}

\section{Article history:}

Received 24 May 2013

Received in revised form 4 July 2013

Accepted 10 July 2013

Available online 20 July 2013

\section{Keywords:}

Tuberculosis

TLR-2

Lipokel

MPT83

Cutinase-like protein1-6

Powdered pulmonary vaccine

\begin{abstract}
A B S T R A C T
Despite considerable research efforts towards effective treatments, tuberculosis (TB) remains a staggering burden on global health. Suitably formulated sub-unit vaccines offer potential as safe and effective generators of protective immunity. The Mycobacterium tuberculosis antigens, cutinase-like proteins (Culp) 1 and 6 and MPT83, were conjugated directly to the novel adjuvant Lipokel (Lipotek Pty Ltd), a TLR2 ligand that delivers antigen to immune cells in a self-adjuvanting context. Protein-Lipokel complexes were formulated as dry powders for pulmonary delivery directly to the lungs of mice by intra-tracheal insufflation, leading to recruitment of neutrophils and antigen presenting cell populations to the lungs at $72 \mathrm{~h}$, that persisted at 7 days post immunisation. Significant increases in the frequency of activated dendritic cells were observed in the mediastinal lymph node (MLN) at 1 and 4 weeks after homologous boosting with protein-Lipokel vaccine. This was associated with the increased recruitment of effector CD4 ${ }^{+}$ and $\mathrm{CD}^{+}$T-lymphocytes to the MLN and systemic antigen-specific, IFN- $\gamma$ producing T-lymphocyte and IgG responses. Notably, pulmonary immunisation with either Culp1-6-Lipokel or MPT83-Lipokel powder vaccines generated protective responses in the lungs against aerosol $M$. tuberculosis challenge. The successful combination of TLR2-targeting and dry powder vaccine formulation, together with important practical benefits, offers potential for pulmonary vaccination against $M$. tuberculosis.
\end{abstract}

(C) 2013 Elsevier Ltd. All rights reserved.

\section{Introduction}

Tuberculosis (TB) represents a staggering burden on global health, with 8.7 million new cases and 1.4 million deaths reported in 2012 alone [1]. The ability of Mycobacterium tuberculosis to persist as a latent infection has allowed the colonisation of one third of the world's population [2]. The only licensed vaccine for TB, Mycobacterium bovis bacille Calmette-Guérin (BCG), is the most widely used vaccine globally and reduces severe neonatal and childhood forms of TB. Protection against adolescent and adult pulmonary TB, however, varies from $0-80 \%$ in different trials [3], and as a live vaccine, BCG possesses significant safety concerns for immunocompromised subjects [2]. Suitably formulated, subunit vaccines offer potential as safe and effective generators of

\footnotetext{
* Corresponding author. Tel.: +61 29515 5210; fax: +61 293513968 . E-mail address: warwick.britton@sydney.edu.au (W.J. Britton).

1 Present address: School of Chemistry and Molecular Biosciences and Australian Infectious Disease Research Centre, University of Queensland, Brisbane, QLD 4072, Australia.
}

immunity to $\mathrm{TB}$, and can be used either alone or to boost immunity in those that have previously received BCG [4]. A number of viral vectored $[5,6]$ or protein-based sub-unit vaccines for TB have entered clinical trials [7-11], however in a recent trial, MVA85A failed to increase protection in infants [5].

The use of pulmonary delivery in TB vaccination is an increasingly appealing strategy. Pulmonary vaccination follows the natural primary route of human infection with $M$. tuberculosis, and may therefore best mimic the induction of a local immune response in the pulmonary mucosa followed by systemic immunity [12]. The surface area of the lung exposes greater regions of lymphoid tissue to antigen than compared with the nasal mucosa, or sub-cutaneous and intra-muscular routes of vaccine administration. Few enzymes or proteases exist at the air-lung interface and the thin epithelial membrane permits efficient absorption of inhaled peptides [13]. Enhanced T-lymphocyte memory has been demonstrated at sites of mucosal priming, with memory T-lymphocytes retained at sites first exposed to antigen [14]. Suitably formulated, dry powder vaccines are able to reach deep within the lungs, and take advantage of the phagocytic activity of alveolar antigen presenting cells (APC) that engulf small-sized particulates [14]. They also possess a 
number of practical advantages as a needleless method of immunisation, with reduced cost of delivery. If suitably formulated in a stable form, they can be easily stored and transported without a cold-chain [15].

The availability of adjuvants capable of stimulating a T-helper 1 (Th1) type immune response is critical for the development of protective TB vaccines [16]. Adjuvants that activate dendritic cells (DC) via Toll-like receptors (TLR) induce a potent Th1 and cytotoxic T lymphocyte $(\mathrm{CTL})$ response, without causing unwanted systemic inflammatory responses [17]. Monophosphoryl lipid A (MPLA) and imiquimod, TLR4 and TLR7 agonists respectively, are the only TLR agonists currently approved for human therapeutic use [18]. Several pattern recognition receptors (PRRs) are involved in host detection of $M$. tuberculosis during pulmonary infection, including the TLRs 2, 4 and 9, utilising the downstream signalling molecule MyD88 [19,20]. Human patients with TLR2 polymorphisms demonstrate increased susceptibility to $M$. tuberculosis infection, while TLR2 ${ }^{-1-}$ mice are unable to mount optimal immune responses against mycobacteria [21].

Targeting antigen to TLR2 expressing cells in the lungs, such as monocytes, neutrophils and alveolar epithelial cells, may stimulate chemokine and cytokine release [21-23]. Simultaneous antigen targeting and activation of DCs is important for the induction of effective Th1 responses [24]. Direct conjugation of peptides to TLR ligands [25-27] promotes antigen accumulation in DCs in the draining lymph node following immunisation [28]. TLR2 ligand stimulation recruits neutrophils and APCs to the immunisation site and migration of DCs to the draining lymph nodes where Th1 responses are initiated [21]. Nevertheless, direct conjugation of whole bacterial protein and TLR agonists has not been examined.

M. tuberculosis antigens, the cutinase-like proteins (CuLPs) 1 and 6 [29-31] and MPT83 [32] are recognised during human TB and stimulate protective immunity against murine $M$. tuberculosis infection. While the individual antigens Culp1 and Culp6 induce protective efficacy when delivered as DNA and protein vaccines in mice, we have also demonstrated additional protective benefits by immunising with a fusion of the two proteins [31]. Therefore in this study, Culp1-6 fusion or MPT83 proteins were conjugated to the novel adjuvant Lipokel (Lipotek Pty Ltd), which is comprised of the lipid moiety Pam2Cys, a TLR2 ligand, linked to the chelating entity 3NTA (Fig. 1A). In the presence of $\mathrm{Ni}^{2+}$, His-tagged protein antigens complex to the 3NTA moiety linked to the TLR2 ligand, and so provide an activation signal to APCs as antigen is encountered. Culp1-6 fusion or MPT83 were conjugated to Lipokel and spray dried to produce an inhalable powder vaccine for pulmonary delivery. Both vaccines were immunogenic and conferred protection against murine $M$. tuberculosis infection. These formulations represent a novel approach to the development of easily administrable and protective vaccines against $\mathrm{TB}$.

\section{Materials and methods}

\subsection{Mice}

Six to eight week old female C57BL/6 mice were obtained from the Animal Resources Centre (Perth, W.A.) and housed under SPF conditions in the Centenary Institute animal facility. All murine experiments were conducted with approval of the University of Sydney Animal Ethics Committee.

\subsection{Bacterial strains and growth conditions}

Escherichia coli was grown in Luria-Bertani (LB) broth or agar at $37^{\circ} \mathrm{C}$, and supplemented with ampicillin $(100 \mu \mathrm{g} / \mathrm{ml})$ or kanamycin $(50 \mu \mathrm{g} / \mathrm{ml}$ ) as necessary. M. tuberculosis H37Rv (ATCC 27294) and
A

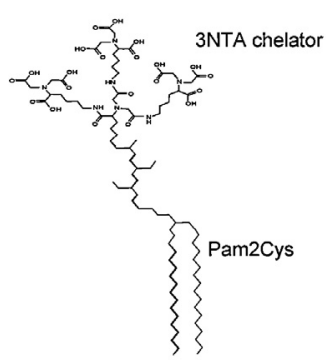

B

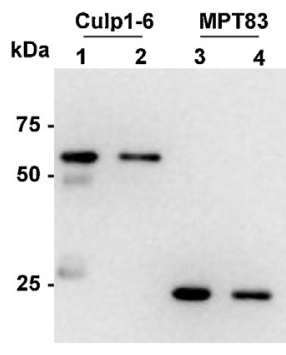

C

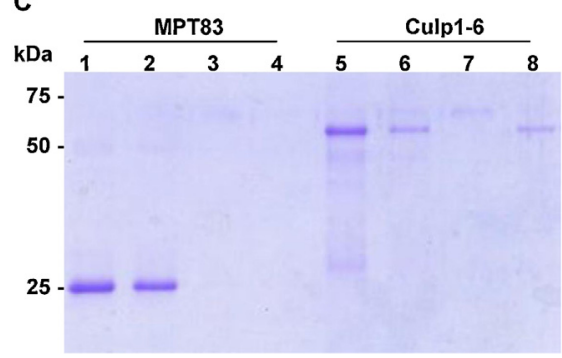

Fig. 1. Conjugation of recombinant proteins Culp1-6 and MPT83 to Lipokel and assessment of inhalable powder vaccines. Purified His-tagged proteins were conjugated to Lipokel in the presence of $\mathrm{Ni}^{2+}$, followed by removal of unconjugated protein via IMAC depletion. Protein-Lipokel complexes were spray dried with mannitol as an excipient, such that a $1 \% \mathrm{w} / \mathrm{w}$ final protein concentration was achieved. (A) Lipokel adjuvant, structure comprises a Pam2Cys molecule conjugated to a 3NTA moiety, capable of binding His-tagged proteins in the presence of $\mathrm{Ni}^{2+}$. (B) Validation of desired protein concentration within powder vaccines via western blotting, as detected by nickel-HRP. Lanes 1 and 3, purified protein $(200 \mu \mathrm{g} / \mathrm{ml})$; lanes 2 and 4 , reconstituted protein-Lipokel powder vaccine $(20 \mathrm{mg} / \mathrm{ml})$. (C) Validation of proteinLipokel complexing within spray dried vaccines. Reconstituted vaccines underwent IMAC depletion to separate complexed and uncomplexed protein, and the fractions were analysed by Coomassie Blue stained SDS-PAGE. Lanes 1 and 5, reconstituted protein-Lipokel vaccine; lanes 2 and 6, complexed fraction of protein-Lipokel powder; lanes 3 and 7, wash of $\mathrm{Co}^{2+}$ resin bed; lanes 4 and 8, uncomplexed fraction of protein-Lipokel powder.

M. bovis BCG Pasteur were cultured in Middlebrook 7H9 (Difco) broth supplemented with albumin-dextrose-catalase (ADC; $10 \%$ $\mathrm{v} / \mathrm{v})$, glycerol $(0.2 \% \mathrm{v} / \mathrm{v})$ and Tween- $80(0.05 \% \mathrm{v} / \mathrm{v})$ at $37^{\circ} \mathrm{C}$. To enumerate $M$. tuberculosis and $M$. bovis, cultures were plated onto Middlebrook 7H11 (Difco) agar, supplemented with oleic-acidalbumin-dextrose catalase (OADC; $10 \% \mathrm{v} / \mathrm{v})$, glycerol $(0.5 \% \mathrm{v} / \mathrm{v})$ and amphotericin $(20 \mu \mathrm{g} / \mathrm{ml})$, and incubated at $37^{\circ} \mathrm{C}$ for 21 days.

\subsection{Construction of plasmid vectors}

The Culp1-6 expression vector pES6 [31] encodes a fusion of the genes Culp1 (Rv1984c) and 6 (Rv3802c) with an N-terminal Histag, expressed by the E. coli expression vector pET-19b (Novagen, Madison, WI). An MPT83 expression vector was constructed utilising the complete coding sequence of mpt83 (Rv2873). The gene was amplified from H37Rv genomic DNA using the forward primer $5^{\prime}$-CATATGATCAACGTTCAGGCC-3' and the reverse primer 5' - AAGCTTCTGTGCCGGGGGCATC-3', and blunt ligated into an intermediate vector (pCR-Blunt II-TOPO; Invitrogen, CA) to form pAT1. The gene was excised using NdeI and HindIII (NEB, Ipswich, MA) and ligated into the digested expression vector pET22b (Novagen) carrying a C-terminal His-tag, to form pAT2.

\subsection{Recombinant protein expression and purification}

Recombinant protein was produced as previously described [31]. Briefly, chemically competent E. coli BL21 (DE3) were transformed with the appropriate protein expression vector, cultured overnight, subcultured and grown until mid-log phase, at which time protein expression was induced with IPTG $(0.5 \mathrm{mM})$. The cells 
were pelleted, resuspended in lysis-solubilisation buffer ( $8 \mathrm{M}$ urea, $50 \mathrm{mM}$ Tris $\mathrm{pH} 7.5,300 \mathrm{mM} \mathrm{NaCl}$ ) and incubated overnight on a rolling circle at $4{ }^{\circ} \mathrm{C}$. Insoluble cell debris was removed via centrifugation. The His-tagged recombinant proteins were purified by $\mathrm{a} \mathrm{Co}^{2+}$ charged, immobilised metal affinity chromatography (IMAC) system (Talon, Clontech), dialysed against $50 \mathrm{mM}$ Tris ( $\mathrm{pH} 7.5$ ), and concentrated with a centrifuge filter (Amicon Ultra-15, MWCO $10 \mathrm{kDa}$, Millipore). Purity was confirmed via SDS-PAGE.

\subsection{SDS-PAGE and western blotting}

Protein samples, along with a molecular weight standard (Bio-Rad, CA), were separated on a two phase $4 \% / 12 \%$ SDSpolyacrylamide stacking/resolving gel and visualised by Coomassie blue staining. For western blotting, proteins were transferred to PVDF, and the membrane blocked with BSA ( $1 \% \mathrm{w} / \mathrm{v}$ in TBST) before His-tagged proteins were detected with nickel-conjugated horseradish peroxidase $(1: 20,000)$ (nickel-HRP His-detect; KPL, Inc., MD). Blots were developed with SuperSignal West Pico Chemiluminescent substrate (Thermo, MA) and visualised using a 4000MM Kodak Image Station.

\subsection{Protein-Lipokel complexing}

Lipokel (Lipotek Pty Ltd) was prepared from powder at $1 \mathrm{mg} / \mathrm{ml}$ in ultra-pure sterile distilled water and sonicated for $30 \mathrm{~s}$. Lipokel was combined with $\mathrm{NiSO}_{4}$ at a molar ratio of 1:2.65 and incubated on a rolling circle for $1 \mathrm{~h}$ at RT (room temperature, $22^{\circ} \mathrm{c}$ ) to allow binding of $\mathrm{Ni}^{2+}$ to the 3NTA moiety of Lipokel. Lipokel-Ni was then added to recombinant His-tagged protein Culp1-6 or MPT83 in a molar ratio of $2: 1$, and similarly incubated for $2 \mathrm{~h}$ to allow formation of the complexes. Uncomplexed protein was removed with a $\mathrm{Co}^{2+}$ IMAC column, and the Lipokel-protein complexes collected. In order to assess the efficiency of complexing, the quantity of protein retained (uncomplexed) on the $\mathrm{Co}^{2+}$ resin bed was eluted, assessed by BCA assay and confirmed by SDS-PAGE.

\subsection{Production of dry powder vaccines}

Pulmonary vaccines were prepared by spray drying (B-290 Mini Spray Dryer, Büchi, Flawil, Switzerland). Protein-Lipokel complex was combined with the excipient mannitol in $10 \mathrm{~mL}$ deionised water, to achieve a final concentration of $1 \% \mathrm{w} / \mathrm{w}$ protein in the spray dried powder. An adjuvant-only control was similarly prepared, utilising an equivalent amount of Lipokel. The spray dryer was operated in open sucking mode with settings as follows: feed rate $1 \mathrm{~mL} / \mathrm{min}$, inlet temperature $50^{\circ} \mathrm{C}$, aspirator $100 \%\left(38 \mathrm{~m}^{3} / \mathrm{h}\right)$ and atomiser $65 \mathrm{mbar}(700-800 \mathrm{~L} / \mathrm{h})$. The collected powders were stored at RT in a desiccator, at an atmosphere of $<20 \%$ humidity.

\subsection{Immunisation of animals}

Pulmonary vaccinations were delivered with a dry powder insufflator (DP-4M, Penn Century, Philadelphia) fitted with a manual air pump (AP-1; Penn Century). The insufflator was primed by passing through spray dried mannitol, prior to loading with $\sim 1 \mathrm{mg}$ vaccine or Lipokel only control. Mice receiving pulmonary vaccines were anaesthetised by intra-peritoneal (IP) injection of ketamine/xylazine solution $(90 / 10 \mathrm{mg} / \mathrm{kg}$ ) and secured on an intubation platform (MIP; Penn Century, Inc). The trachea was visualised with an illuminated otoscope (MDS, w/6" lens; Hallowell EMC, Pittsfield, MA) and the mouse intubated with the preloaded insufflator until the tip was immediately adjacent to the first bifurcation of the trachea. A quick succession of ten $250 \mu$ l puffs of air was delivered, aerosolising the powder and delivering the vaccine directly to the surface of the lungs. The insufflator was removed and weighed to confirm the quantity of vaccine delivered. The mouse was rested on a heating pad for 20 min before IP injection of atipamezole $(0.0125 \mathrm{mg} / \mathrm{mouse})$, and monitored until self-righting and mobile. Mice receiving BCG were anaesthetised with isofluorane vapour and immunised with a single sub-cutaneous injection on the back with $5 \times 10^{5}$ CFU BCG-Pasteur.

\subsection{Cellular responses to immunisation}

Flow cytometry was utilised to examine the cellular response to pulmonary immunisation. Innate responses in the lung were assessed at days 3 and 7 after a single vaccination. Antibody panel utilised was anti-CD11b APCCy7 (M1/70; BD Biosciences, San Jose, CA), anti-CD11c PECy7 (HL3; BD Biosciences), anti-CD86 FITC (GL1; BD Biosciences), anti-MHCII APC (M5/114.15.2; eBioscience, San Diego, CA), anti-Ly6G PerCPCy5.5 (1A8; Biolegend, San Diego, CA), anti-B220 PE (RA3-6B2; eBioscience), anti-CD45.2 PB (104; Biolegend) and live/dead fixable blue dead cell stain (Invitrogen). Innate and adaptive responses were analysed in the mediastinal lymph node (MLN) and spleen of mice given three immunisations two weeks apart, at days 7 and 28 after final immunisation. The antibody panel utilised, in addition to anti-CD11c, anti-CD86, anti-MHCII and live/dead stain listed above, included anti-CD3 PerCP (145-2C11; BD Biosciences), anti-CD4 AF700 (RM45; BD Biosciences), anti-CD8 APC Cy7 (53-6.7; BD Biosciences), anti-CD62L e450 (MEL-14; eBioscience) and anti-CD44 PE (IM7; Biolegend). Single-cell suspensions were prepared from the MLN, spleen, or lung. Lung tissue was digested with collagenase type 4197 (50U/ml; Freehold NJ) and DNAse I (13 $\mu \mathrm{g} / \mathrm{ml}$; Sigma) at $37^{\circ} \mathrm{C}$ for $45 \mathrm{~min}$ prior to homogenisation and multiple filtration steps. Erythrocytes were removed using ACK lysis buffer in the case of spleen or lung homogenates. After blocking with anti-mouse CD16/CD32 (2.4G2; BD Biosciences), $3 \times 10^{6}$ cells were incubated with appropriate antibody mix to stain for surface markers. Cells were fixed overnight in $5 \%$ neutral buffered formalin at $4{ }^{\circ} \mathrm{C}$, and the data acquired using a FACS LSRII or LSRFortessa flow analyser (BD Biosciences).

\subsection{Murine IFN- $\gamma$ T-lymphocyte responses}

Mice were sacrificed at four weeks following two vaccinations two weeks apart, and antigen-specific IFN- $\gamma$ secreting splenocytes enumerated by ELIspot as previously described [32]. Purified rCulp1-6 or rMPT83 protein $(10 \mu \mathrm{g} / \mathrm{ml})$ were used as antigens, with Concanavalin A (Con A) $(3 \mu \mathrm{g} / \mathrm{ml})$ or media alone as controls. The total production of IFN- $\gamma$ following in vitro stimulation of splenocytes was measured by ELISA. $2 \times 10^{5}$ splenocytes were stimulated for $72 \mathrm{~h}$ with either rCulp1-6 or rMPT83 protein $(10 \mu \mathrm{g} / \mathrm{ml})$, with the above controls. Culture supernatants were analysed for IFN- $\gamma$ release by ELISA as previously described [32].

\subsection{Murine serum IgG responses}

Serum anti-Culp1-6 or anti-MPT83 IgG titres of vaccinated mice were determined by ELISA. Plates were coated with either rCulp1-6 or rMPT83 $(2 \mu \mathrm{g} / \mathrm{ml})$, followed by blocking with $3 \% \mathrm{w} / \mathrm{v}$ BSA. Sera samples were diluted $1: 100$ and added to the ELISA plate for $1 \mathrm{~h}$ at $37^{\circ} \mathrm{C}$. Following washing, the plates were incubated with alkaline phosphatase-conjugated, goat anti-mouse IgG antibody (1:2000; Sigma). The assay was developed via the addition of substrate and the absorbance read at $405 \mathrm{~nm}$.

\subsection{Experimental M. tuberculosis infection}

Six weeks following the final pulmonary immunisation, mice were challenged with $M$. tuberculosis by low-dose aerosol infection 
$(100 \mathrm{CFU})$ in an inhalation exposure system (Glas-Col, Terre Haute, IN). Four weeks later, bacterial loads in the lungs and spleen were determined by plating serial dilutions of the tissue homogenate, incubated at $37^{\circ} \mathrm{C}$ for 21 days.

\subsection{Statistical analysis}

Differences in cellular responses, serum IgG, or bacterial counts were analysed using one-way or two-way analysis of variance (ANOVA), followed by Bonferroni post hoc analysis to control for multiple comparisons. The differences in IFN- $\gamma$ responses between immunised and control mice were analysed using an unpaired Student's $t$-test with Welch's correction. Statistical analysis was performed using the GraphPad Prism 6 software (GraphPad Software, La Jolla, CA). Differences between groups were considered significant when the $P$ values were $\leq 0.05$.

\section{Results}

\subsection{Assessment of protein-Lipokel conjugate inhalable powder vaccines}

M. tuberculosis antigens Culp1-6 and MPT83 were expressed with His-tags as recombinant proteins in E. coli, solubilised, purified and refolded by dialysis. Culp1-6 was identified by SDS-PAGE and western blotting to have a relative molecular mass of approximately $55 \mathrm{kDa}$ and MPT83 approximately $25 \mathrm{kDa}$. Protein-Lipokel conjugates were produced and spray dried with mannitol to achieve a $1 \% \mathrm{w} / \mathrm{w}$ protein concentration. In order to assess whether degradation had occurred during the spray drying process, powder vaccines were reconstituted and integrity confirmed by either western blotting or SDS-PAGE (Fig. 1B and C).

\subsection{Early innate immune responses generated in the lung after pulmonary vaccination}

In order to assess early innate immune responses generated by the powder vaccines, $1 \mathrm{mg}$ Lipokel control or Culp1-6-Lipokel vaccine was delivered intra-tracheally to C57BL/6 mice $(n=5)$. Three or 7 days after a single pulmonary immunisation, whole lungs were processed for flow cytometry analysis focusing on myeloid lineage subsets (Fig. 2A). At day 3 post vaccination, the ${\text { Ly } 6 G^{+}}^{+} \mathrm{CD}_{11 \mathrm{~b}^{+}}$neutrophil population was significantly increased in both the Lipokel only and Culp1-6-Lipokel groups. By day 7, this response was reduced, but remained significantly increased in the Culp1-6-Lipokel group (Fig. 2B). Ly6G $^{-}$B220- ${ }^{-}$populations were gated into 5 groups according to the levels of CD11b and CD11c expression. At day 3, CD11 $b^{\text {hi }}$ CD $11 c^{\text {int-hi }}$ (myeloid DCs), CD11b $b^{\text {hi }}$ CD11 $\mathrm{c}^{-}$(recruited monocytes) and $\mathrm{CD} 11 \mathrm{~b}^{\text {int }} \mathrm{CD} 11 \mathrm{c}^{\text {lo }}$ (pulmonary macrophages) subsets with a $\mathrm{CD}^{+} 6^{+} \mathrm{MHCII}{ }^{\text {hi }}$ phenotype were all significantly increased in both the Lipokel only and Culp1-6-Lipokel groups. By day 7 these responses had reduced by approximately 10 -fold, however they remained significantly higher in the Culp16-Lipokel group (Fig. 2C). Therefore pulmonary immunisation with the protein-Lipokel vaccine led to a sustained increase in the frequency of activated DCs and macrophages in the lungs.

\subsection{Sustained DC and T-lymphocyte responses to pulmonary vaccination}

In order to assess whether pulmonary vaccination with proteinLipokel conjugate induced sustained responses, C57BL/6 mice received three homologous immunisations at two-weekly intervals with Lipokel only, Culp1-6-Lipokel or MPT83-Lipokel. Flow cytometry was utilised to enumerate the $\mathrm{CD}^{-} \mathrm{CD} 11 \mathrm{c}^{+} \mathrm{CD}^{-} 6^{+} \mathrm{MHCII}^{\mathrm{hi}}$ population of activated DCs in the MLN at days 7 and 28 after the

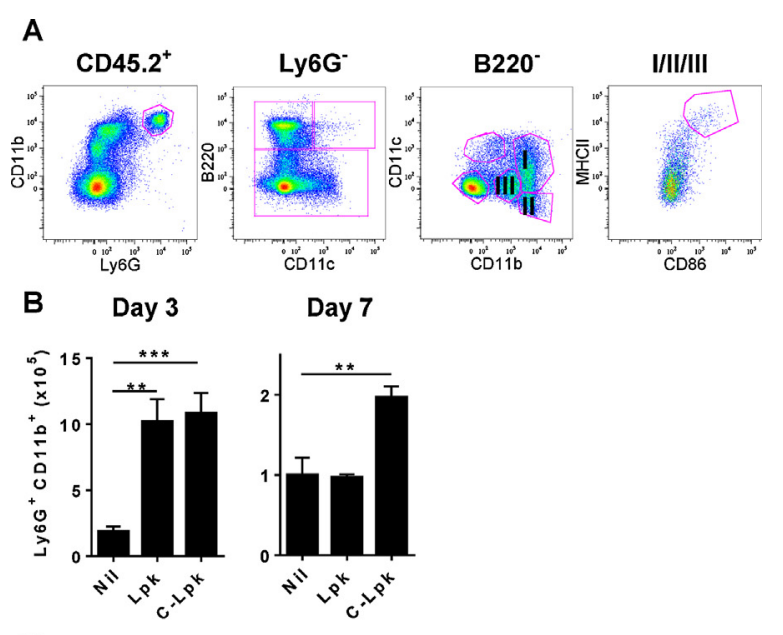

C

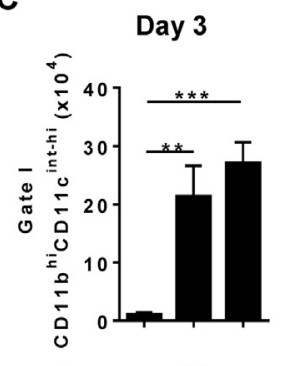

Day 7
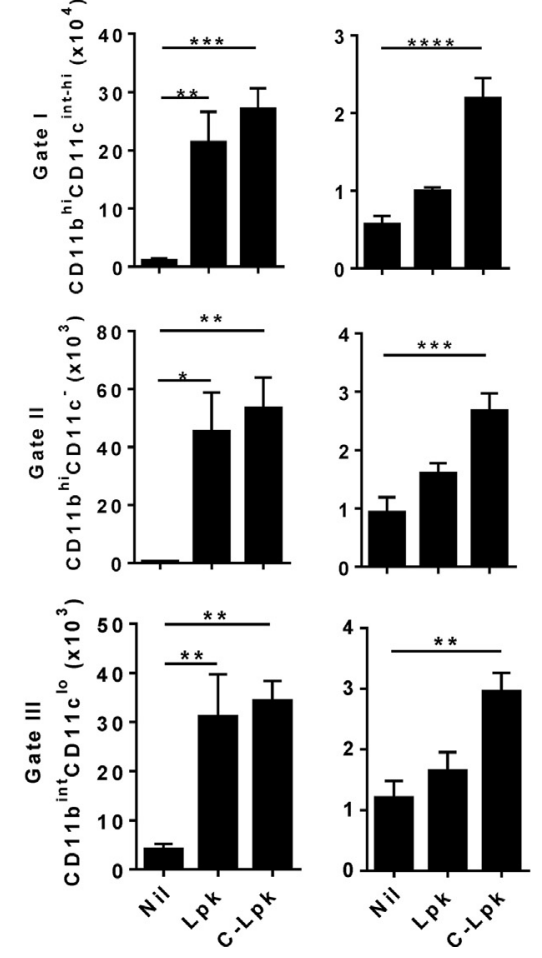

Fig. 2. Early induction of neutrophils and APC subsets in the lungs after pulmonary vaccination. $\mathrm{C} 57 \mathrm{BL} / 6$ mice $(n=5)$ were immunised with $10 \mu \mathrm{g}$ of conjugated Culp16 protein, or an equivalent amount of Lipokel, by intra-tracheal insufflation of $1 \mathrm{mg}$ powder. At day 3 or 7 post single immunisation, innate responses in the lungs were determined via flow cytometry. (A) Initial gates were set based on FSC and SSC characteristics to exclude debris, RBC, and doublets. Dead cells were excluded, then the CD $45.2^{+}$population determined. The subsequent gating strategy is shown. (B) The $\mathrm{Ly}_{6 \mathrm{G}} \mathrm{CD}^{+} 11 \mathrm{~b}^{+}$neutrophil population was increased in number in both treatment groups at day 3, but remained increased after 7 days only in the Culp1-6-Lipokel group. (C) Ly6G $^{-}$B220- populations were gated based on expression of CD11b and CD11c, and were further analysed based on CD86 ${ }^{+} \mathrm{MHCII}^{\mathrm{hi}}$ expression. At day 3 in both treatment groups, there were increases in the frequency of (I) CD11 $\mathrm{b}^{\text {hi }} \mathrm{CD} 11 \mathrm{c}^{\text {int-hi }}$ (myeloid DCs), (II) CD11b $\mathrm{b}^{\text {hi }}$ CD11 $\mathrm{c}^{-}$(recruited monocytes) and (III) $\mathrm{CD} 11 \mathrm{~b}^{\text {int }} \mathrm{CD} 11 \mathrm{c}^{\mathrm{lo}}$ (pulmonary macrophages) that were also $\mathrm{CD}^{+} 6^{+} \mathrm{MHCII}^{\mathrm{hi}}$. These populations remained increased at day 7 in the Culp1-6Lipokel group only. The data are the means $\pm S E M$, with the statistical significance determined by ANOVA and Bonferroni post hoc comparison $\left({ }^{*} p<0.05,{ }^{* *} p<0.01\right.$, $\left.{ }^{* * *} p<0.001,{ }^{* * * *} p<0.0001\right)$.

final immunisation (Fig. 3A). At these time points, Lipokel alone did not induce a significant increase in DCs. By contrast, Culp16-Lipokel induced a significant increase in activated DCs in the MLN compared to unvaccinated controls at day 7 , however this 
A
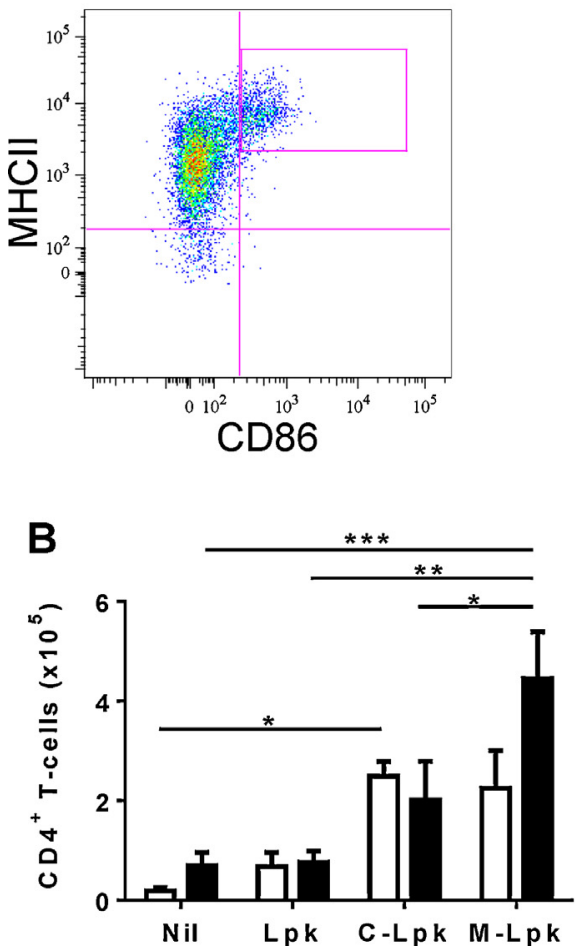
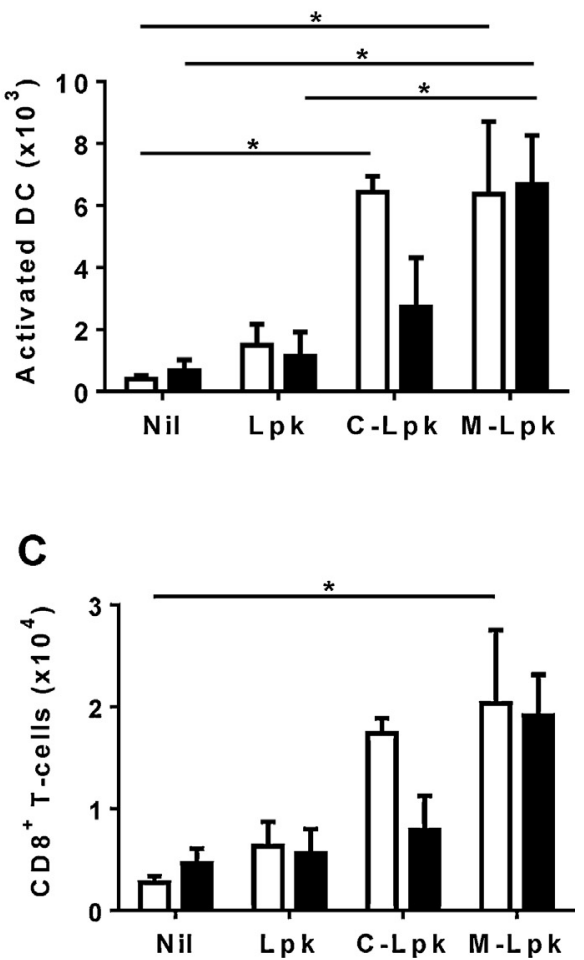

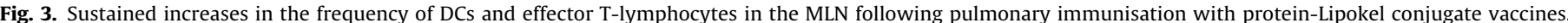

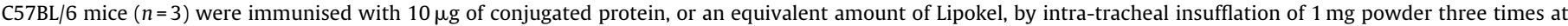

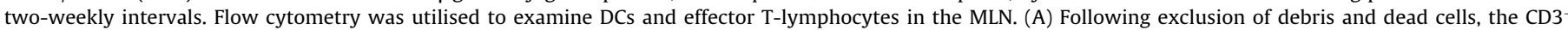

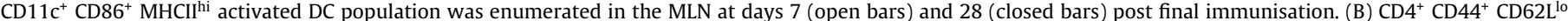

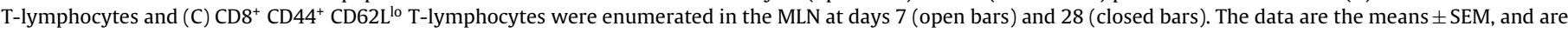
representative of repeat experiments. Statistical significance was determined by ANOVA with Bonferroni post hoc comparison $\left({ }^{*} p<0.05,{ }^{* *} p<0.01,{ }^{* * *} p<0.001\right)$.

response waned by day 28. Immunisation with MPT83-Lipokel, however, resulted in a sustained DC response to the vaccine at both days 7 and 28 (Fig. 3A). The expansion of CD44+ CD62 L ${ }^{\text {lo }}$ activated effector T-lymphocyte populations were examined in the MLN and spleen at the same time points. In the MLN at day 7 , Culp1-6-Lipokel induced a significant increase in CD4 ${ }^{+}$effector Tlymphocytes that persisted at day 28 (Fig. 3B). There was a similar trend for increased expansion of $\mathrm{CD}^{+}$effector T-lymphocytes at day 7 (Fig. 3C). Interestingly, MPT83-Lipokel generated significantly higher numbers of $\mathrm{CD} 4^{+}$effector T-lymphocytes in the MLN at day 28 , (Fig. 3B), while CD8 ${ }^{+}$effector T-lymphocytes were increased at day 7 and 28 (Fig. 3C). In the spleen, MPT83-Lipokel immunised mice demonstrated increased numbers of $\mathrm{CD}^{+}$and $\mathrm{CD}^{+}$effector T-Lymphocytes at day 28 (data not shown).

\subsection{Pulmonary vaccination with protein-Lipokel conjugate induces systemic IFN- $\gamma$ secreting T-lymphocytes and $\operatorname{Ig} G$ production}

In order to assess antigen-specific responses to pulmonary vaccination, IFN- $\gamma$-secreting T-lymphocyte responses were analysed by ELIspot and ELISA using splenocytes from vaccinated mice. Significantly higher numbers of antigen-specific IFN- $\gamma$ secreting cells were observed in the spleens of both Culp1-6-Lipokel (Fig. 4A) and MPT83-Lipokel (Fig. 4B) immunised groups. After $72 \mathrm{~h}$ of stimulation with the respective antigens, splenocytes from Culp16 -Lipokel vaccinated mice secreted significantly increased IFN- $\gamma$ compared to Lipokel alone (Fig. 4C), and there was a similar trend for IFN- $\gamma$ production by splenocytes from MPT83-Lipokel vaccinated mice (Fig. 4D).
In addition, both Culp1-6-Lipokel and MPT83-Lipokel stimulated significant antigen-specific IgG responses in the sera, as measured by ELISA (Fig. 5A and B).

\subsection{Protective efficacy of protein-Lipokel vaccine conjugates}

Finally, the protective efficacy of Culp1-6-Lipokel and MPT83Lipokel were assessed in a mouse model of low-dose aerosol M. tuberculosis infection. C57BL/6 mice received three homologous pulmonary immunisations two weeks apart, with either $1 \mathrm{mg}$ Lipokel control or protein-Lipokel vaccine powder, and were rested for 6 weeks. Mice were challenged by aerosol infection with $M$. tuberculosis (100 CFU), and the bacterial loads in the lung and spleen enumerated four weeks later. In the lung, both Culp1-6-Lipokel and MPT83-Lipokel conjugated powdered vaccines provided a significant protective effect when compared to unvaccinated controls (Fig. 6A). No protective effect was generated in response to the pulmonary vaccines in the spleen (Fig. 6B).

\section{Discussion}

The continued burden of TB indicates the urgent need for novel, safe, efficacious and easily deliverable vaccines against TB [33]. While BCG is likely to be retained because of its protective effects in infants, subunit vaccines offer the advantage of increased safety over BCG in immune-compromised individuals, and the potential to boost the effects of BCG in adults. Direct pulmonary delivery of viral vectors presenting $M$. tuberculosis antigens provides some protective efficacy, but repeat use is limited by the immune response to the virus backbone [34]. To our knowledge, this is the first study to 
A

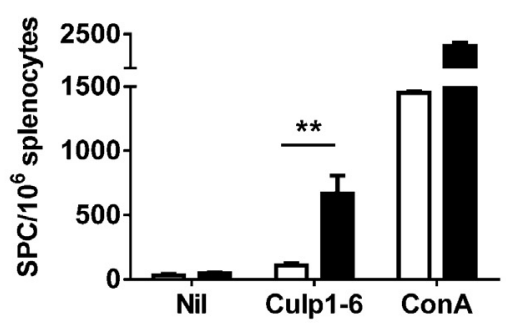

C

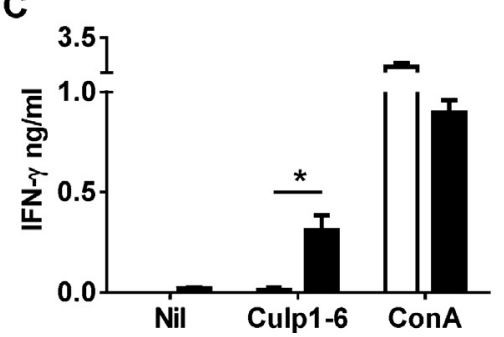

B

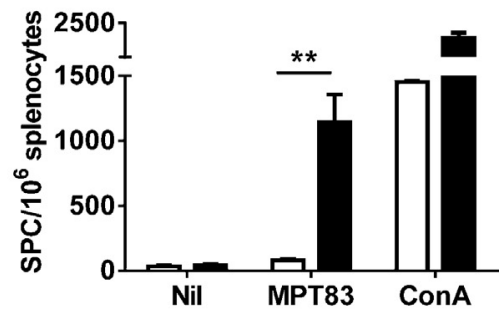

D

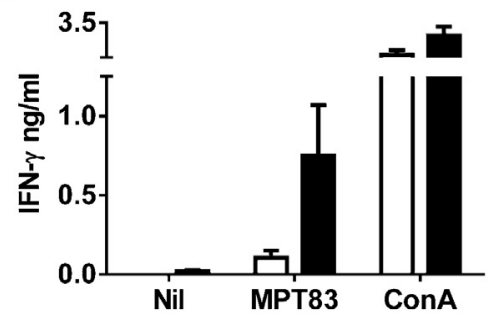

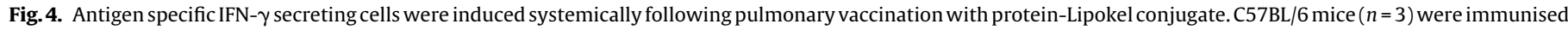

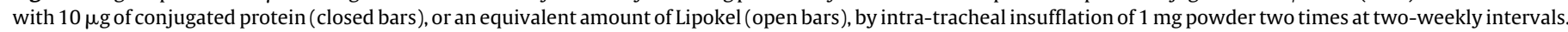

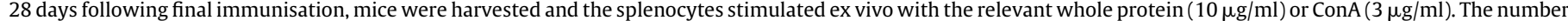

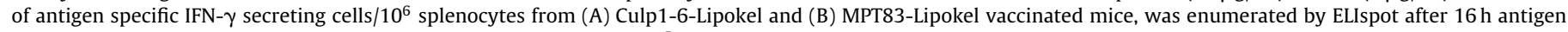

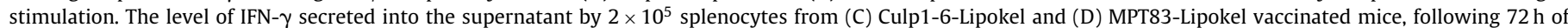

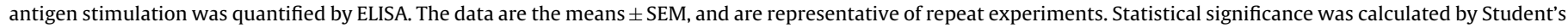
$t$-test with Welch's correction $\left({ }^{*} p<0.05,{ }^{* *} p<0.01\right)$.

A

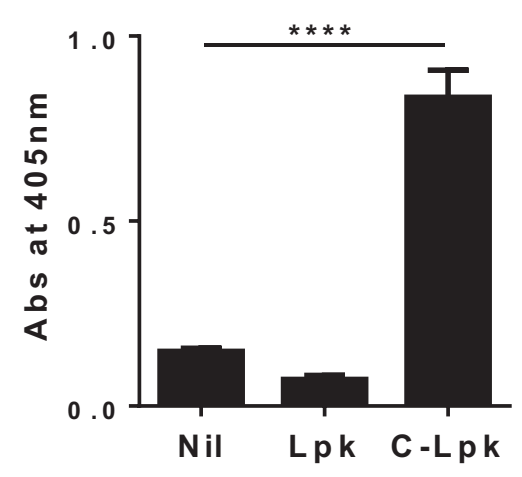

B

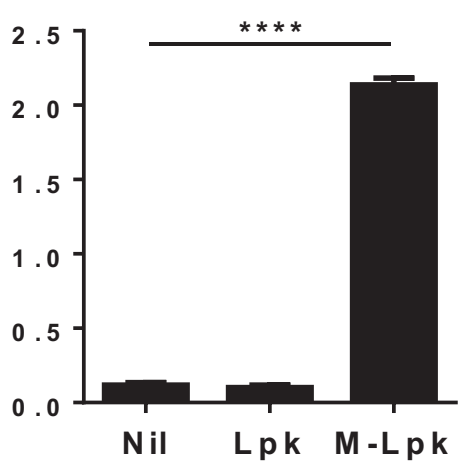

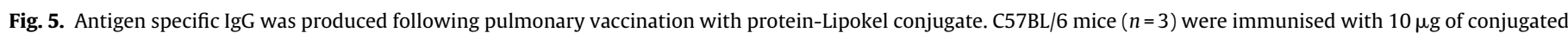

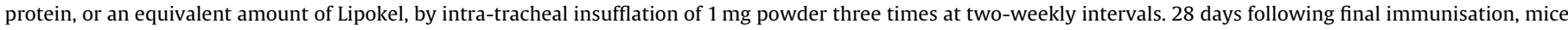

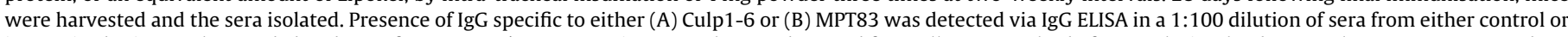

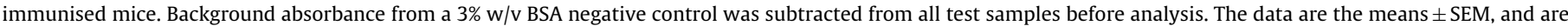
representative of repeat experiments. Statistical significance was determined by ANOVA with Bonferroni post hoc comparison $(* * * * p<0.001)$.

demonstrate protection from virulent $M$. tuberculosis aerosol challenge by homologous boosting with a powdered pulmonary protein vaccine.

After conjugating the two protein antigens to Lipokel, the TLR2targeted vaccines were successfully formulated as aerosolisable powders by spray drying. Spray drying technology is a readily scalable process that offers the ability to alter vaccine formulation in order to optimise aerosol delivery to the deep lung. Antigen distribution within the respiratory tract has a major effect on the type of immune response generated. In the case of inhaled influenza vaccines, targeting the deep lung induced a local shift of the cellular immune response towards an optimum Th1 phenotype [35]. This technology has been utilised to develop a number of powdered vaccines of varying types, including whole live bacteria, viral vaccines and sub-unit proteins [14]. These dry powder vaccines are stable over a prolonged period and, in the case of live vaccines, such as BCG and influenza, viability is maintained [36-38].

Mannitol was chosen as an excipient for the powdered vaccines in this study, as it is approved for inhaled medicinal use [39]. In addition, unlike other polyol excipients, such as lactose, mannitol is crystalline and relatively stable when spray dried [40]. Importantly, no protein degradation was detected by western blotting of the powder vaccines (Fig. 1B), and all the MPT83 remained conjugated to Lipokel after spray drying, storage at RT and reconstitution (Fig. 1C). It appeared that a portion of the Culp1-6 protein was unconjugated in the powder vaccine (Fig. 1C). This uncoupling may occur on a protein-protein basis, depending on the stability of the protein and availability of the His-tag. Alternatively, it is possible that not all unconjugated Culp1-6 was removed prior to spray drying. Production of recombinant protein with a His-tag at both the $\mathrm{N}$ and $\mathrm{C}$ terminus may maximise binding opportunities with Lipokel 
A

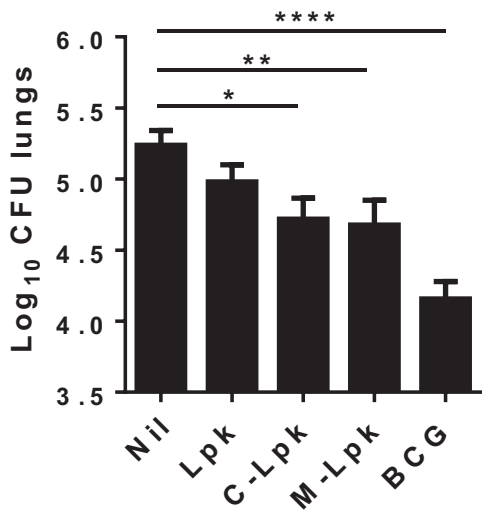

B

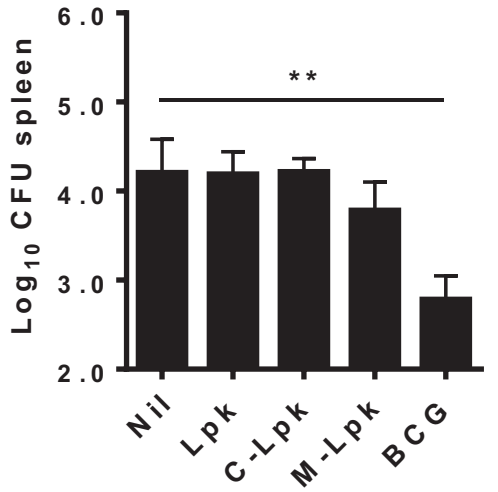

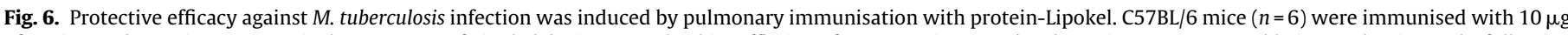

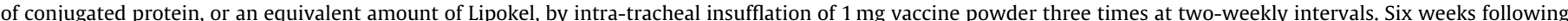

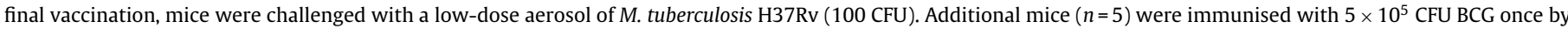

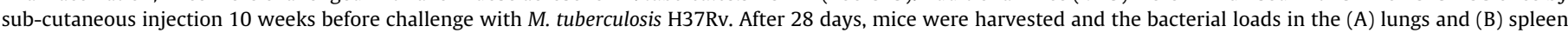

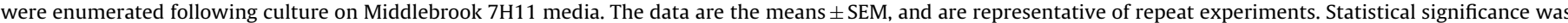
determined by ANOVA with Bonferroni post hoc comparison to unvaccinated control $\left({ }^{*} p<0.05,{ }^{* *} p<0.01\right.$, $\left.{ }^{* * * *} p<0.0001\right)$.

and reduce the possibility of non-adjuvanted protein being delivered into the lung. This strategy may also provide an opportunity to conjugate multiple Lipokel molecules per protein, and this may increase the adjuvant effect.

The innate immune responses generated in the lung by Lipokel vaccines demonstrate the potential of Pam2Cys to act as a pulmonary mucosal adjuvant. TLR2 is expressed on multiple cell types in the pulmonary environment, in particular on monocytes and neutrophils, and is significantly upregulated on pulmonary epithelial cells during $M$. tuberculosis infection [21-23]. TLR2 signalling plays a major role in regulating neutrophil migration across activated endothelia, possibly by increasing cell surface expression of chemokine receptors and activating CD11b/CD18 [21]. Stimulation of TLR2 results in the secretion of CXCL8, a neutrophil, monocyte and T-lymphocyte chemoattractant that is found at high levels in the plasma and broncho-alveolar lavage fluid of TB patients, as well as CCL2, a potent chemoattractant and activator of monocytes and CD4 ${ }^{+}$T-lymphocytes [21]. TLR2 stimulation also leads to production of TNF and IL-6, which is critical for the initial protective IFN $\gamma$ response during $M$. tuberculosis infection [21].

In this study, significant influx of neutrophils, myeloid DCs, monocytes and pulmonary macrophages occurred in the lungs by day 3 after a single pulmonary immunisation with either Lipokel or Culp1-6-Lipokel conjugate. These cell populations remained increased at day 7 only in the protein-Lipokel immunised mice (Fig. 2). This sustained effect may be due to the induction of Tlymphocyte responses to the protein antigen, with associated IFN- $\gamma$ release maintaining the enhanced innate immune responses. At day 7 and 28 after homologous boosting, both DCs and effector $\mathrm{CD}^{+}$and $\mathrm{CD}^{+}$T-lymphocytes were increased in the MLN only after vaccination with the protein-Lipokel conjugate (Fig. 3). This was accompanied by systemic induction of antigen-specific, IFN- $\gamma$ producing T-lymphocytes (Fig. 4), as well as production of antigenspecific IgG antibodies (Fig. 5).

Lipokel offers a unique method of directly conjugating protein antigens to Pam2Cys, thereby targeting antigen to APCs whilst also providing an activation signal. Antigen-adjuvant complexing may limit the systemic release of potentially harmful pro-inflammatory cytokines induced after delivery of alternative soluble immuno-modulators [24,41]. Furthermore, Lipokel produced no visible adverse effects, whereas formulation with more reactogenic compounds may create concerns for pulmonary delivery. Induction of cellular immunity via DC stimulation may also enhance the control of existing infection, which is a desirable property for a new TB vaccine [42].

This study demonstrates the immunogenicity and protective efficacy of novel powdered pulmonary vaccines, utilising the TLR2 ligand Pam2Cys directly conjugated to recombinant $M$. tuberculosis proteins. The Lipokel adjuvant technology offers potential for use with a variety of recombinant His-tagged proteins, with possible applications for vaccines against other respiratory pathogens. In addition to the potential immunological benefits, dry powder pulmonary sub-unit vaccines offer practical advantages for community based immunisation. They can be readily mass produced, stored and transported without the need for a cold-chain, and may be easily administered via the use of a dry powder inhaler. There is further potential to formulate powder vaccines utilising additional adjuvants to stimulate other PRRs expressed in the pulmonary environment. Therefore these TLR2-targeted, pulmonary dry powder vaccines represent a novel approach for the prevention of TB.

\section{Financial support}

National and Medical Research Council of Australia, the NSW Government through its infrastructure grant to the Centenary Institute, and the Federal Government through the Biotechnology Innovation Fund (BIF) which supported the development of Lipokel. AST JGYC and ERS are recipients of Australian Postgraduate Awards.

\section{Conflicts of interest}

IA is an employee of Lipotek Pty Ltd.

\section{Acknowledgements}

The authors thank Lipotek Pty Ltd for the provision of the adjuvant, Dr M Flórido, Dr BM Saunders, Ms A Pang, Ms C Gilles, and Mr P Reynolds for guidance and technical assistance, and Dr GC Feng for reviewing the manuscript. 


\section{References}

[1] WHO. Global Tuberculosis Control: WHO Report 2012. Geneva: World Health Organization; 2012.

[2] Kaufmann SHE. Future vaccination strategies against tuberculosis: thinking outside the box. Immunity 2010;33:567-77.

[3] Andersen P, Doherty TM. The success and failure of BCG-implications for a novel tuberculosis vaccine. Nat Rev Micro 2005;3:656-62.

[4] Dey B, Jain R, Gupta UD, Katoch VM, Ramanathan VD, Tyagi AK. A booster vaccine expressing a latency-associated antigen augments BCG induced immunity and confers enhanced protection against tuberculosis. PLoS One 2011;6:e23360.

[5] Tameris MD, Hatherill M, Landry BS, Scriba TJ, Snowden MA, Lockhart S, et al. Safety and efficacy of MVA85A, a new tuberculosis vaccine, in infants previously vaccinated with BCG: a randomised, placebo-controlled phase $2 \mathrm{~b}$ trial. Lancet 2013;1:60177-84

[6] Hoft DF, Blazevic A, Stanley J, Landry B, Sizemore D, Kpamegan E, et al. A recombinant adenovirus expressing immunodominant TB antigens can significantly enhance BCG-induced human immunity. Vaccine 2012;30:2098-108.

[7] Spertini F, Audran R, Lurati F, Ofori-Anyinam O, Zysset F, Vandepapelière P, et al. The candidate tuberculosis vaccine Mtb72F/AS02 in PPD positive adults: a randomized controlled phase I/II study. Tuberculosis 2013;93:179-88.

[8] van Dissel JT, Soonawala D, Joosten SA, Prins C, Arend SM, Bang P, et al. Ag85BESAT-6 adjuvanted with IC $31^{\circledR}$ promotes strong and long-lived Mycobacterium tuberculosis specific T cell responses in volunteers with previous BCG vaccination or tuberculosis infection. Vaccine 2011;29:2100-9.

[9] Lindenstrøm T, Agger EM, Korsholm KS, Darrah PA, Aagaard C, Seder RA et al. Tuberculosis subunit vaccination provides long-term protective immunity characterized by multifunctional CD4 memory T cells. J Immunol 2009;182:8047-55.

[10] Billeskov R, Elvang TT, Andersen PL, Dietrich J. The HyVac4 subunit vaccine efficiently boosts BCG-primed anti-mycobacterial protective immunity. PLoS One 2012;7:e39909.

[11] Lin PL, Dietrich J, Tan E, Abalos RM, Burgos J, Bigbee C, et al. The multistage vaccine $\mathrm{H} 56$ boosts the effects of BCG to protect cynomolgus macaques against active tuberculosis and reactivation of latent Mycobacterium tuberculosis infection. J Clin Invest 2012;122:303-14.

[12] Todoroff J, Ucakar B, Inglese M, Vandermarliere S, Fillee C, Renauld JC, et al. Targeting the deep lungs, Poloxamer 407 and a CpG oligonucleotide optimize immune responses to Mycobacterium tuberculosis antigen 85A following pulmonary delivery. Eur J Pharm Biopharm 2013;84:40-8.

[13] Tomoda K, Makino K. Effects of lung surfactants on rifampicin release rate from monodisperse rifampicin-loaded PLGA microspheres. Colloids Surf B Biointer 2007;55:115-24.

[14] Lu D, Hickey AJ. Pulmonary vaccine delivery. Expert Rev Vacc 2007;6:213.

[15] Kunda NK, Somavarapu S, Gordon SB, Hutcheon GA, Saleem IY. Nanocarriers targeting dendritic cells for pulmonary vaccine delivery. Pharm Res 2013;30:325-41.

[16] O'Garra A, Redford PS, McNab FW, Bloom CI, Wilkinson RJ, Berry MPR. The immune response in tuberculosis. Ann Rev Immunol 2013;31:475-527.

[17] Hedayat M, Takeda K, Rezaei N. Prophylactic and therapeutic implications of Toll-like receptor ligands. Med Res Rev 2012;32:294-325.

[18] Vacchelli E, Galluzzi L, Eggermont A, Fridman WH, Galon J, Sautès-Fridman C, et al. Trial watch: FDA-approved Toll-like receptor agonists for cancer therapy. Oncoimmunology 2012;1:894-907.

[19] Fremond CM, Yeremeev V, Nicolle DM, Jacobs M, Quesniaux VF, Ryffel B. Fatal Mycobacterium tuberculosis infection despite adaptive immune response in the absence of MyD88. J Clin Invest 2004;114:1790-9.

[20] Juffermans NP, Florquin S, Camoglio L, Verbon A, Kolk AH, Speelman P, et al. Interleukin-1 signaling is essential for host defense during murine pulmonary tuberculosis. J Infect Dis 2000;182:902-8.

[21] Andersson M, Lutay N, Hallgren O, Westergren-Thorsson G, Svensson M Godaly G. Mycobacterium bovis bacilli Calmette-Guerin regulates leukocyte recruitment by modulating alveolar inflammatory responses. Innate Immun 2012;18:531-40.
[22] Hertz CJ, Wu Q, Porter EM, Zhang YJ, Weismüller K-H, Godowski PJ, et al. Activation of Toll-like receptor 2 on human tracheobronchial epithelial cells induces the antimicrobial peptide human $\beta$ defensin-2. J Immunol 2003;171:6820-6.

[23] Li Y, Wang Y, Liu X. The role of airway epithelial cells in response to mycobacteria infection. Clin Dev Immunol 2012:11.

[24] Kamath AT, Mastelic B, Christensen D, Rochat A-F, Agger EM, Pinschewer $\mathrm{DD}$, et al. Synchronization of dendritic cell activation and antigen exposure is required for the induction of Th1/Th17 responses. J Immunol 2012;188:4828-37.

[25] Huleatt JW, Jacobs AR, Tang J, Desai P, Kopp EB, Huang Y, et al. Vaccination with recombinant fusion proteins incorporating Toll-like receptor ligands induces rapid cellular and humoral immunity. Vaccine 2007;25:763-75.

[26] Blander JM, Medzhitov R. Toll-dependent selection of microbial antigens for presentation by dendritic cells. Nature 2006;440:808-12.

[27] Khan S, Bijker MS, Weterings JJ, Tanke HJ, Adema GJ, van Hall T, et al. Distinct uptake mechanisms but similar intracellular processing of two different Toll-like receptor ligand-peptide conjugates in dendritic cells. J Biol Chem 2007;282:21145-59.

[28] Kastenmüller K, Wille-Reece U, Lindsay RWB, Trager LR, Darrah PA, Flynn BJ, et al. Protective T cell immunity in mice following protein-TLR7/8 agonistconjugate immunization requires aggregation, type I IFN, and multiple DC subsets. J Clin Invest 2011;121:1782-96.

[29] West NP, Wozniak TM, Valenzuela J, Feng CG, Sher A, Ribeiro JMC, et al. Immunological diversity within a family of cutinase-like proteins of Mycobacterium tuberculosis. Vaccine 2008;26:3853-9.

[30] West NP, Chow FME, Randall EJ, Wu J, Chen J, Ribeiro JMC, et al. Cutinaselike proteins of Mycobacterium tuberculosis: characterization of their variable enzymatic functions and active site identification. FASEB J 2009;23:1694-704.

[31] Shanahan ER, Pinto R, Triccas JA, Britton WJ, West NP. Cutinase-like protein-6 of Mycobacterium tuberculosis is recognised in tuberculosis patients and protects mice against pulmonary infection as a single and fusion protein vaccine. Vaccine 2010;28:1341-6.

[32] Kao FF, Mahmuda S, Pinto R, Triccas JA, West NP, Britton WJ. The secreted lipoprotein, MPT83, of Mycobacterium tuberculosis is recognized during human tuberculosis and stimulates protective immunity in mice. PLoS One 2012; 7:e34991.

[33] Dye C, Glaziou P, Floyd K, Raviglione M. Prospects for tuberculosis elimination. Annu Rev Public Health 2013;34:271-86.

[34] White AD, Sibley L, Dennis MJ, Gooch K, Betts G, Edwards N, et al. Evaluation of the safety and immunogenicity of a candidate tuberculosis vaccine, MVA85A, delivered by aerosol to the lungs of macaques. Clin Vaccine Immunol 2013;20:663-72.

[35] Minne A, Louahed J, Mehauden S, Baras B, Renauld J-C, Vanbever R. The delivery site of a monovalent influenza vaccine within the respiratory tract impacts on the immune response. Immunology 2007;122:316-25.

[36] Garcia-Contreras L, Wong YL, Muttil P, Padilla D, Sadoff J, DeRousse J, et al. Immunization by a bacterial aerosol. Proc Natl Acad Sci USA 2008;105:4656-60.

[37] Morello M, Krone CL, Dickerson S, Howerth E, Germishuizen WA, Wong Y$\mathrm{L}$, et al. Dry-powder pulmonary insufflation in the mouse for application to vaccine or drug studies. Tuberculosis 2009;89:371-7.

[38] Saluja V, Amorij JP, Kapteyn JC, de Boer AH, Frijlink HW, Hinrichs WLJ. A comparison between spray drying and spray freeze drying to produce an influenza subunit vaccine powder for inhalation. J Cont Release 2010;144:127-33.

[39] Hurt K, Bilton D. Inhaled mannitol for the treatment of cystic fibrosis. Expert Rev Respir Med 2012;6:19-26.

[40] Naini V, Byron PR, Phillips EM. Physicochemical stability of crystalline sugars and their spray-dried forms: dependence upon relative humidity and suitability for use in powder inhalers. Drug Dev Ind Pharm 1998;24:895-909.

[41] Lingnau K, Egyed A, Schellack C, Mattner F, Buschle M, Schmidt W. Polyl-arginine synergizes with oligodeoxynucleotides containing CpG-motifs (CPG-ODN) for enhanced and prolonged immune responses and prevents the CPG-ODN-induced systemic release of pro-inflammatory cytokines. Vaccine 2002;20:3498-508.

[42] Palucka K, Banchereau J, Mellman I. Designing vaccines based on biology of human dendritic cell subsets. Immunity 2010;33:464-78. 